\title{
Mesurer les liaisons entre espèces dans un groupe de relevés
}

\author{
Ph. Daget ${ }^{1}$ A. Gaston ${ }^{2}$
}

\section{Mots-clés}

Pâturage - Zone tropicale - Mesure M éthode - Végétation - Tchad.

\section{Résumé}

Deux types de liaison interspécifique sont distingués, la contingence fondée sur la prise en compte des probabilités totales et l'association fondée sur celle des probabilités conditionnelles. U ne méthode d'analyse de l'association est présentée et illustrée par un cas concret tchadien.

\section{- CONTINGEN CE EN TRE ESPECES}

Depuis les débuts de la phytosociologie quantitative, la caractérisation de l'association des espèces a fait l'objet de préoccupations des biométriciens (4). Ils ont, la plupart du temps, abordé cette mesure par le coefficient de contingence (2) ou coefficient de corrélation de point.

Soit deux espèces, présentes ensemble ou séparément dans un lot de $\mathrm{N}$ relevés, et soit a le nombre de relevés où ces deux espèces sont présentes ensemble, b celui des relevés qui ne contient que la première, c celui des relevés qui ne contient que la seconde et $\mathrm{d}$ le nombre de relevés qui ne contient aucune des deux espèces; le coefficient de contingence s'écrit $(1,2)$ :

$$
c=\frac{a d-b c}{\sqrt{(a+b)(a+c)(c+d)(b+d)}}
$$

Lorsqu'il y a un très grand nombre de relevés, ou lorsqu'il s'agit de mesurer l'association d'espèces peu fréquentes, le poids des relevés «vides » (du moins en ce qui concerne ces deux espèces) devient prépondérant et produit un biais important dans la caractérisation cherchée. C'est pourquoi Godron et coll. (3) ont proposé de calculer la contingence en faisant abstraction de $\mathrm{d}$.

Une des qualités importantes de la contingence est d'être réversible ; la liaison de l'espèce $A$ avec l'espèce $B$ est la même que celle de l'espèce $B$ avec l'espèce A. Pour l'agro-pastoraliste ou l'écologue sur le terrain, elle correspondrait à la réflexion suivante : « Si, sur le terrain, il m'arrivait de rencontrer l'espèce A, alors la probabilité de rencontrer l'espèce $\mathrm{B}$ serait celle-ci. »

1. CIRAD-EMVT/CNRS, Campus international de Baillarguet, BP 5035, 34032 Montpellier Cedex 1, France

2. Laboratoire d'agronomie, INRA-INA,78850 Thivernal-Grignon, France

\section{ASSO CIATION ENTRE ESPECES}

\section{Principe de la caractérisation}

Il en va autrement sur le terrain. En effet, l'observateur de terrain n'a que faire de suppositions ; il voit une espèce particulière en arrivant dans la station qu'il veut caractériser ou analyser ; il la voit parce qu'elle est dominante, ou parce qu'elle a des fleurs très voyantes, ou encore parce que son port est très caractéristique et la réflexion qu'il se fait est la suivante : «L'espèce A est présente ici, donc j'ai des chances de trouver les espèces $\mathrm{B}$ et $\mathrm{D} »$. Il ne se pose même pas de question sur l'espèce $C$, elle n'est pas associée à $\mathrm{A}$. L'expérience peut conduire certains observateurs soigneux à une très grande précision dans la prévision du cortège qu'ils vont rencontrer. Cette expérience mène aussi à de nouvelles questions : «Comment se fait-il que l'espèce F ne soit pas présente ici, c'est anormal... » Anomalie qui traduit généralement un début d'évolution vers des faciès différents (début de dégradation, de surexploitation, première influence d'une sécheresse, ou l'inverse, selon les cas) et provoque une analyse particulière du phénomène pressenti.

Le problème de la contingence est en définitive un examen des probabilités totales (1) comparant, pour les aléas i et $\mathrm{j}$ :

$$
p(i, j) \text { à } p(i) \cdot p(j)
$$

tandis que celui de l'association, qui est envisagé ici, se rapporte aux probabilités conditionnelles (1), comparant :

$$
p(i \mid j) \text { à } p(i)
$$

Cette comparaison sera faite aisément au moyen du test $\chi^{2}$ (6) dans la mesure où les effectifs traités sont suffisamment importants, et par un test t avec report à une table s'ils sont faibles (1) :

$$
\chi^{2}=\frac{(\mathrm{O}-\mathrm{Np})^{2}}{\mathrm{Npq}}
$$


où $\mathrm{N}$ est le nombre de relevés contenant l'espèce de référence, $\mathrm{p}$ la fréquence relative de l'espèce qui lui est comparée dans l'ensemble de l'inventaire et $\mathrm{O}$ le nombre de relevés comportant à la fois l'espèce de référence et celle qui est examinée, avec $q=1$ - $p$. Avec les conventions de Dagnélie (2) utilisées dans le paragraphe précédent, on peut écrire :

$$
\chi^{2}=\frac{(a d-b c)^{2}}{(a+c)(a+b)(c+d)}
$$

Cette écriture, ne contenant pas les arrondis des fréquences relatives, donne des résultats plus précis que la précédente.

L'utilisation de ce critère et de la fréquence relative des espèces dans les relevés contenant l'espèce de référence permet de classer l'association de ces espèces en cinq catégories :

- association absolue : la fréquence relative de l'espèce comparée dans les relevés extraits est 1 ;

- association presque certaine : le $\chi^{2}$ est supérieur à 6,63 (valeur liminaire au seuil de 0,99);

- association très probable : il est compris entre 6,63 et 3,84 (valeur liminaire au seuil de 0,95$)$;

- association probable : il est compris entre 3,84 et 1,32 (valeur liminaire au seuil de 0,75$)$;

- pas d'association : il est inférieur à 1,32.

\section{Exemple}

Le tableau I représente un extrait tiré de la base FLOTROP du CIRAD-EMVT (7) et correspond aux 20 premières espèces de 41 relevés faits en novembre 1974 par A. Gaston, alors agropastoraliste à l'IEMVT, dans la cuvette du lac Tchad. Sur les originaux de ses relevés, il a noté que certains d'entre eux correspondaient à des steppes à Hyphaene thebaica, d'autres à des steppes à Acacias, d'autres à des jachères ou à des types de végétation divers.

Prenons le cas d'Hyphaene thebaica. Le tableau II réunit, en présence/absence, les 10 relevés où cette espèce a été enregistrée et les espèces trouvées au moins une fois dans ces relevés ; il comporte aussi le nombre d'occurences de chacune de ces espèces dans l'ensemble des 41 relevés, dans les 10 retenus ici, et les fréquences relatives correspondantes.

Parmi ces espèces, Cenchrus biflorus a donc été trouvé 12 fois dans l'inventaire et 7 fois en présence d'Hyphaene thebaica. Le $\chi^{2}$ qui mesure la liaison entre ces deux espèces est donc:

$$
\chi^{2}=\frac{(7-10 \times 0,29)^{2}}{10 \times 0,29 \times 0,71}=\frac{4,1^{2}}{2,06}=8,16
$$

C'est une valeur nettement supérieure aux valeurs liminaires de 3,84 et 6,63 pour les seuils de 0,95 et 0,99 . En définitive, il est hautement probable que Cenchrus biflorus soit lié avec Hyphaene thebaica ou, en d'autres termes, dans une steppe à Hyphaene thebaica, la présence de Cenchrus biflorus est hautement probable ; ceci, naturellement, dans la mesure où les relevés de base constituent bien un échantillon représentatif de la situation régionale.

Sur la base de cet échantillon, il est possible d'appliquer le critère ci-dessus autant de fois qu'il y a d'espèces. Ainsi :

- Dans la steppe à Hyphaene thebaica, il y a presque certainement : Alysicarpus ovalifolius, Aristida funiculata, Aristida mutabilis, Brachiaria distichophylla, Cenchrus biflorus, Commiphora africana, Dactyloctenium aegyptium, Monsonia senegalensis, Salvadora persica, Schoenefeldia gracilis, Sclerocarya birrea, Tribulus terrestris; et sûrement : Eragrostis tremula, Hyperthelia dissoluta, Maerua crassifolia.

\section{Tableau I}

Extrait de la base FLOTRO P pour les 20 premières espèces de 41 des relevés de $A$. G aston dans la cuvette du Tchad en novembre 1974

$\begin{array}{ll}0005 & \text { Acacia albida } \\ 0011 & \text { Acacia nilotica } \\ 0017 & \text { Acacia senegal } \\ 0018 & \text { Acacia seyal } \\ 0019 & \text { Acacia sieberiana } \\ 0023 & \text { Achyranthes aspera } \\ 0027 & \text { Alysicarpus ovalifolius } \\ 0077 & \text { Aristida funiculata } \\ 0077 & \text { Aristida longiflora } \\ 0080 & \text { Aristida mutabilis } \\ 0093 & \text { Balanites aegyptiaca } \\ 0095 & \text { Bauhinia rufescens } \\ 0115 & \text { Boscia senegalensis } \\ 0118 & \text { Brachiaria distichophylla } \\ 0122 & \text { Brachiaria ramosa } \\ 0123 & \text { Brachiaria xantholeuca } \\ 0131 & \text { Cadaba farinosa } \\ 0133 & \text { Calotropis procera } \\ 0148 & \text { Cassia tora } \\ 0153 & \text { Cenchrus biflorus }\end{array}$

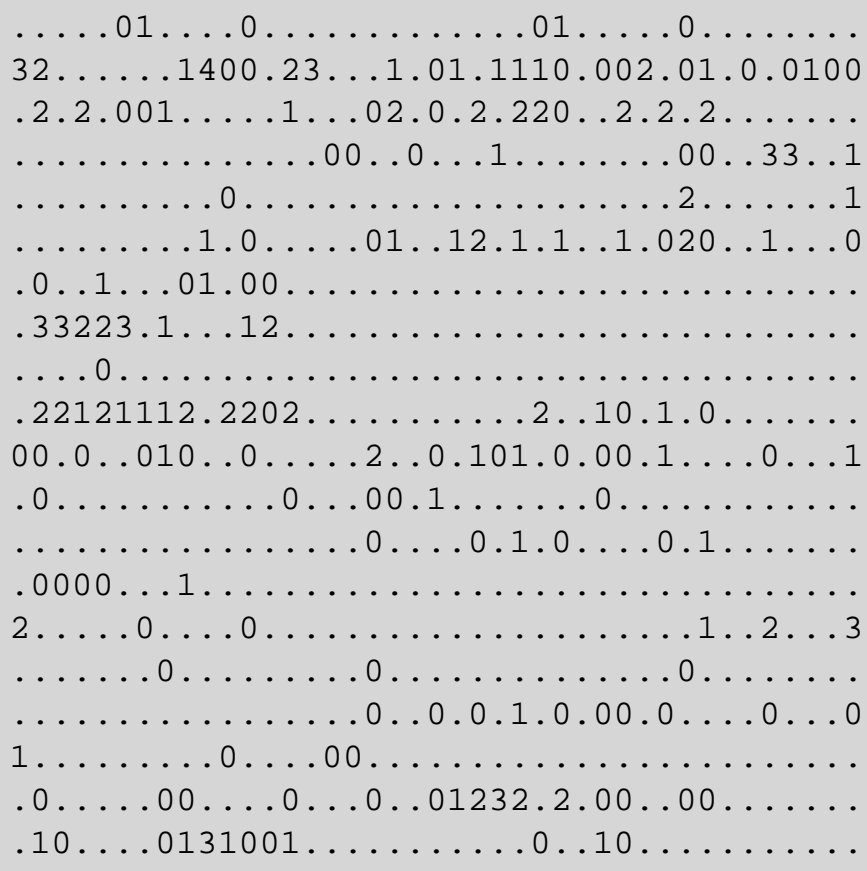


Tableau II

Matrice en présence/absence des espèces rencontrées au moins une fois avec $\mathrm{H}$ yphaene thebaica dans les relevés précédents, fréquences absolues et relatives

\begin{tabular}{|c|c|c|c|c|c|}
\hline & & Ft & Fh & FRt & Frh \\
\hline Acacia albida & 0001100100 & 6 & 3 & 0,14 & 0,3 \\
\hline Acacia nilotica & 1000001101 & 25 & 4 & 0,6 & 0,4 \\
\hline Acacia senegal & 1001110001 & 16 & 5 & 0,39 & 0,5 \\
\hline Acacia seyal & 0000000000 & 9 & 0 & 0,21 & 0 \\
\hline Acacia sieberiana & 0000000000 & 3 & 0 & 0,07 & 0 \\
\hline Achyranthes aspera & 0000000100 & 14 & 1 & 0,34 & 0,1 \\
\hline Alysicarpus ovalifolius & 1010001110 & 6 & 5 & 0,14 & 0,5 \\
\hline Aristida funiculata & 1111010110 & 8 & 7 & 0,19 & 0,7 \\
\hline Aristida longiflora & 0010000000 & 1 & 1 & 0,02 & 0,1 \\
\hline Aristida mutabilis & 1111111111 & 17 & 10 & 0,41 & 1 \\
\hline Balanites aegyptiaca & 1000111100 & 18 & 5 & 0,43 & 0,5 \\
\hline Bauhinia rufescens & 1000000001 & 6 & 2 & 0,14 & 0,2 \\
\hline Boscia senegalensis & 0000000000 & 6 & 0 & 0,14 & 0 \\
\hline Brachiaria distichophylla & 1110001000 & 5 & 4 & 0,12 & 0,4 \\
\hline Brachiaria ramosa & 0000100100 & 6 & 2 & 0,14 & 0,2 \\
\hline Brachiaria xantholeuca & 0000010000 & 3 & 1 & 0,07 & 0,1 \\
\hline Cadaba farinosa & 0000000000 & 10 & 0 & 0,24 & 0 \\
\hline Calotropis procera & 0000000000 & 4 & 0 & 0,09 & 0 \\
\hline Cassia obtusifolia & 1000011001 & 15 & 4 & 0,36 & 0,4 \\
\hline Cenchrus biflorus & 1100011111 & 12 & 7 & 0,29 & 0,7 \\
\hline Chloris pilosa & 1011111101 & 26 & 8 & 0,63 & 0,8 \\
\hline Commelina forsskalaei & 0010000110 & 5 & 3 & 0,12 & 0,3 \\
\hline Commiphora africana & 0111100010 & 6 & 5 & 0,14 & 0,5 \\
\hline Dactyloctenium aegyptium & 1111111111 & 23 & 10 & 0,56 & 1 \\
\hline D igitaria horizontalis & 1000010101 & 7 & 4 & 0,17 & 0,4 \\
\hline Echinochloa colona & 0000000000 & 6 & 0 & 0,14 & 0 \\
\hline Eragrostis tremula & 1000101100 & 6 & 4 & 0,14 & 0,4 \\
\hline Hyparrhenia dissoluta & 0011000100 & 4 & 3 & 0,09 & 0,3 \\
\hline Hyphaene thebaica & 1111111111 & 10 & 10 & 0,24 & 1 \\
\hline Ipomoea pestigridis & 0000000001 & 3 & 1 & 0,07 & 0,1 \\
\hline Maerua angolensis & 0000011000 & 3 & 2 & 0,07 & 0,2 \\
\hline Maerua crassifolia & 1100100000 & 4 & 3 & 0,09 & 0,3 \\
\hline Monsonia senegalensis & 0011000010 & 3 & 3 & 0,07 & 0,3 \\
\hline O ryza barthii & 0000000000 & 1 & 0 & 0,02 & 0 \\
\hline Panicum laetum & 0000000000 & 10 & 0 & 0,24 & 0 \\
\hline Panicum maximum & 0000000000 & 5 & 0 & 0,12 & 0 \\
\hline Pennisetum pedicellatum & 0000001101 & 14 & 3 & 0,34 & 0,3 \\
\hline Piliostigma reticulata & 0100100000 & 4 & 2 & 0,09 & 0,2 \\
\hline Salvadora persica & 1100111100 & 6 & 6 & 0,14 & 0,6 \\
\hline Schizachyrium sanguineum & 0000001000 & 1 & 1 & 0,02 & 0,1 \\
\hline Schoenefeldia gracilis & 1111101010 & 8 & 7 & 0,19 & 0,7 \\
\hline Sclerocarya birrea & 0101100100 & 5 & 4 & 0,12 & 0,4 \\
\hline Tribulus terrestris & 1000011001 & 4 & 4 & 0,09 & 0,4 \\
\hline U rochloa trichopus & 0000010000 & 2 & 1 & 0,04 & 0,1 \\
\hline Ximenia americana & 0000000000 & 1 & 0 & 0,02 & 0 \\
\hline Ziziphus mauritiana & 1000010001 & 19 & 3 & 0,46 & 0,3 \\
\hline Zornia glochidiata & 0000000000 & 5 & 0 & 0,12 & 0 \\
\hline
\end{tabular}


- De même, en partant des relevés contenant Cadaba farinosa, il apparaît que dans ces steppes, avec Cadaba farinosa il y a presque certainement : Achyranthes aspera, Balanites aegyptiaca, Boscia senegalensis ; et sûrement : Acacia senegal, Cassia obtusifolia, Ziziphus mauritiana.

- Dans ces steppes, la fin de la matrice dont un extrait est donné dans le tableau I, montrerait qu'avec Ziziphus mauritiana, il y a presque certainement : Acacia senegal ; et sûrement : Cassia obtusifolia.

Il est possible de prendre deux espèces à la fois en faisant la somme booléenne* de leurs présences/absences dans l'ensemble des relevés traités.

Il reste enfin à traiter de gros ensembles de relevés, d'une part, pour que le test utilisé prenne sa pleine validité et, d'autre part, pour voir si et comment l'analyse permet de rejoindre l'expérience de terrain.

\section{CONCLUSION}

L'approche habituelle de la caractérisation des relations interspécifiques porte sur l'étude de l'indépendance entre les espèces et peut s'exprimer par : les probabilités de présence des espèces $A$ et $B$ étant respectivement $\mathrm{pA}$ et $\mathrm{pB}$, la probabilité de trouver à la fois $\mathrm{A}$ et $\mathrm{B}$ est $\mathrm{pA} \times \mathrm{pB}$. C'est la voie principale pour la mise en évidence au laboratoire des associations et autres groupements végétaux (5). La méthode exposée ici des liaisons interspécifiques conditionnelles répond au problème plus réaliste sur le terrain : l'espèce $A$ existant ici (elle est, par exemple, dominante), quelle est la probabilité de trouver aussi B ?

\section{Summary}

D aget Ph., Gaston A. M easuring connections between species in a group of relevés

Two kinds of interspecific connections are discerned, the contingency based on independent probabilities and the association based on conditional probabilities. An association analytical method is presented and illustrated with a Chadian case.

Key words: Grazing - Tropical zone - Measurement - Method Vegetation - Chad.

\section{BIBLIO GRAPHIE}

1. CONOVER W., 1980. Practical nonparametric statistics. Wiley, New York, USA, $493 \mathrm{p}$.

2. DAGNELIE P., 1962. Etude statistique d'une pelouse à Brachipodium ramosum, III. Liaisons interspécifiques. Bull. Serv. Carte phytogéogr., Série $B, 7$ : 99-109; 7 : 149-160.

3. GODRON M., GUILLERM J., ROMAN, F., SABATO-PIZZINI L., 1969. Sur l'interprétation des matrices de coefficients de corrélation en phytosociologie. 0 ecol. Plant., 4 : 15-26.

4. GOUNOT M., 1958. Contribution à l'étude des groupements rudéraux et messicoles de la Tunisie. Ann. Serv. Bot. Agron. Tunisie, 31 : $1-382$.

5. GUINOCHET M., 1974. Phytosociologie. Paris, France, Masson, $226 \mathrm{p}$.

6. SNEDECOR G., COCHRAN W., 1968. Statistical methods. Ames, USA, lowa State Univ. Press, $512 \mathrm{p}$.

7. TACHER G., 1993. Le projet FLOTROP au CIRAD-EMVT. Flotrop info, $1: 1-2$.

Reçu le 30.1.97, accepté le 4.8 .97

\begin{abstract}
* On appelle vecteur booléen, un vecteur qui ne comporte que des 1 et des 0 , donc une suite de présences/absences. La somme booléenne de deux vecteurs booléens de même longueur consiste à leur associer un troisième vecteur booléen, également de même longueur, comportant un 1 à chaque emplacement où l'un des vecteurs initiaux en comporte un, et un 0 là où les deux vecteurs initiaux ont un $0 ;$ la somme booléenne de 111000 et de 001100 est donc 111100 .
\end{abstract}

\section{Resumen}

Daget Ph., G aston A. Medida de las relaciones entre las especies en un grupo de muestras

Se distinguen dos tipos de relación inter específica, la contingencia basada las probabilidades totales y la asociación basada en las probabilidades condicionales. Se ilustra un método de análisis de la asociación mediante un caso chadiano concreto.

Palabras clave: Pastoreo - Zona tropical - Medición - Método Vegetación - Chad. 